\title{
COMPUTING TRANSFER MAPS FROM MAGNETIC FIELD DATA*
}

\author{
M. Venturini ${ }^{\dagger}$ and A. Dragt, \\ Physics Department, University of Maryland, College Park MD 20742
}

\begin{abstract}
In many cases the most accurate information about fields in a magnet comes either from direct measurement (using for example spinning coils) or from a numerical computation done with a 3D electromagnetic code. In this paper we show how this information can be used to compute transfer maps with high accuracy. The resulting transfer maps take into account all effects of real beamline elements including fringe-field and multipole error effects. The method we employ automatically incorporates the smoothing properties of the Laplace Green function. Consequently, it is robust against both measurement and electromagnetic code errors. The method has been implemented in the code MARYLIE as a pair of user-defined routines.
\end{abstract}

\section{INTRODUCTION}

The motion of charged particles through any beam-line element is described by the transfer map $\mathcal{M}$ for that element. Through aberrations of order $(n-1)$ such a map has the Lie representation $[1,2]$

$$
\mathcal{M}=\mathcal{R}_{2} \exp \left(: f_{3}:\right) \exp \left(: f_{4}:\right) \cdots \exp \left(: f_{n}:\right) .
$$

The linear map $\mathcal{R}_{2}$ and the Lie generators $f_{\ell}$ are determined by the equation of motion $\dot{\mathcal{M}}=\mathcal{M}:-H$ : where $H=H_{2}+H_{3}+H_{4}+\cdots$ is the Hamiltonian expressed in terms of deviation variables and expanded in a homogeneous polynomial series. The deviation variable Hamiltonian $H$ is determined in turn by the Hamiltonian $K$. In Cartesian coordinates with $z$ taken as the independent variable, and in the absence of electric fields, $K$ is given by the relation

$K=-\left[p_{t}^{2} / c^{2}-m^{2} c^{2}-\left(p_{x}-q A_{x}\right)^{2}-\left(p_{y}-q A_{y}\right)^{2}\right]^{1 / 2}-q A_{z}$

Here $\boldsymbol{A}$ is the magnetic vector potential. We therefore need a Taylor expansion for the vector potential components $A_{x}$, $A_{y}, A_{z}$ in the deviation variables $x$ and $y$. How can the coefficients of the Taylor expansion for the vector potential be determined from a knowledge of the magnetic field? In this paper we review the method we proposed in [3]. The method uses information about the fields coming from either direct measurement or numerical computation done with a 3D electromagnetic code. It is based on the calculation of Fourier integrals with suitable kernels derived from the Green function of the Laplace equation. Our approach is different from and more accurate than other methods based on numerical differentiation (e.g. [4]). A pleasant feature is relative insensitivity to the presence of noise

\footnotetext{
* Work was supported by the US Department of Energy.

†venturin@physics.umd.edu
}

in the magnetic field data, which makes the method capable of providing accurate computations of high order terms in the desired Taylor expansion. An additional advantage is that it applies, with minor modifications, to both magnet data obtained by numerical computation and measured data found with spinning coils (see [3] for more details).

\section{DETERMINATION OF THE VECTOR POTENTIAL}

In a current-free region the magnetic field $\boldsymbol{B}$ can be described most simply in terms of a scalar potential $\psi$ (with $\boldsymbol{B}=\nabla \psi$ ) obeying the Laplace equation $\nabla^{2} \psi=0$. In cylindrical coordinates the general solution to this equation (that is regular for small $\rho$ ) has the expansion

$$
\psi=\sum_{m=0}^{\infty} \int_{-\infty}^{\infty} d k e^{i k z} I_{m}(k \rho)\left[\hat{b}_{m} \sin m \phi+\hat{a}_{m} \cos m \phi\right],
$$

where the functions $\hat{a}_{m}=\hat{a}_{m}(k)$ and $\hat{b}_{m}=\hat{b}_{m}(k)$ are arbitrary, and $I_{m}$ is the modified Bessel function. This is a "cylindrical multipole" expansion, where $m$ is related to the order of the multipole, and should not be confused with a spherical multipole expansion. The first term on the RHS of (2) describes a purely solenoidal field $(m=0)$. The other terms in the series correspond to the dipole $(m=1)$, quadrupole $(m=2), \cdots$ components. For simplicity we will treat the terms with $m \geq 2$. The solenoidal term requires a separate, but analogous, treatment that entails no new complications. The dipole case is more complicated. In the sometimes restrictive case that the sagitta of the design orbit does not exceed the radius $R$ introduced in Sec. 3, the methods of this paper also apply. However other methods are required if the sagitta is larger. If $\psi$ is given in the form (2), a suitable corresponding vector potential is easily found. Since there is gauge freedom, a possible covenient choice, in the absence of a solenoidal component, is to work in a gauge satisfying $A_{\phi}=0$. Suppose $\psi$ as given by (2) is rewritten in the form

$$
\psi=\sum_{m=1}^{\infty} \psi_{m, s}(\rho, z) \sin m \phi+\psi_{m, c}(\rho, z) \cos m \phi
$$

with

$$
\psi_{m, s}(\rho, z)=\int_{-\infty}^{\infty} d k e^{i k z} I_{m}(k \rho) \hat{b}_{m}(k) .
$$

[ $\psi_{m, c}$ has the same form, with $\hat{a}_{m}(k)$ replacing $\hat{b}_{m}(k)$.] Then it is easily verified that the remaining components of 
the vector potential are given by the relations

$$
\begin{aligned}
& A_{\rho}=\sum_{m=1}^{\infty} \frac{\cos (m \phi)}{m} \rho \frac{\partial}{\partial z} \psi_{m, s}-\frac{\sin (m \phi)}{m} \rho \frac{\partial}{\partial z} \psi_{m, c} \\
& A_{z}=\sum_{m=1}^{\infty}-\frac{\cos (m \phi)}{m} \rho \frac{\partial}{\partial \rho} \psi_{m, s}+\frac{\sin (m \phi)}{m} \rho \frac{\partial}{\partial \rho} \psi_{m, c} .
\end{aligned}
$$

From the two equations above it is clear that finding Taylor expansions for the vector potential components $A_{x}$, $A_{y}$, and $A_{z}$ (what we need) is equivalent to finding Taylor expansions for $\psi_{m, s}$ and $\psi_{m, c}$ in the variable $\rho$. This is easily done by a two-step process: first, we expand the modified Bessel functions $I_{m}(k \rho)$ appearing in (4) as Taylor series in the quantity $(k \rho)$. Doing so produces an expansion in powers of $\rho$ with coefficients that involve integrations over various powers of $k$. Second, we observe that the powers of $k$ can be replaced by multiple differentiation with respect to the variable $z$. The net results of these two steps are the relations $(\alpha=c, s)$

$$
\psi_{m, \alpha}(\rho, z)=\sum_{\ell=0}^{\infty} \frac{(-1)^{\ell} m !}{2^{2 \ell} \ell !(\ell+m) !} C_{m, \alpha}^{[2 \ell]}(z) \rho^{2 \ell+m} .
$$

The index $[2 \ell]$ indicates the $2 \ell$ derivative with respect to the longitudinal variable $z$. The functions $C_{m, \alpha}^{[0]}(z)$ are the generalized on-axis gradients. Note that the generalized gradients depend on the longitudinal variable $z$. For fields produced by long well-made magnets, however, the $z$ dependence will be significant only at the ends. We conclude that the dynamics of a charged particle passing through a region of space occupied by a magnetic field described by the scalar potential (2) is completely determined by a knowledge of the generalized on-axis gradient functions $C_{m, \alpha}^{[0]}(z)$ and their derivatives.

\section{COMPUTATION OF GENERALIZED GRADIENTS FROM FIELD DATA}

Suppose the radial component of the magnetic field $B_{\rho}$ is known, either by measurement or computation, on the surface of some infinitely long cylinder of radius $R$. Moreover, suppose that the field is given in terms of an angular Fourier series,

$$
B_{\rho}=\sum_{m=1}^{\infty} B_{m}(R, z) \sin (m \phi)+A_{m}(R, z) \cos (m \phi) .
$$

It can be shown [3] that the generalized on-axis gradients appearing in the expansion coefficients for the scalar potential (8) can be written as

$$
C_{m, s}^{[n]}(z)=\frac{i^{n}}{2^{m} m !} \frac{1}{\sqrt{2 \pi}} \int_{-\infty}^{\infty} d k e^{i k z} \frac{k^{m+n-1}}{I_{m}^{\prime}(k R)} \tilde{B}_{m}(R, k) .
$$

The expression for $C_{m, c}^{[n]}(z)$ has $\tilde{A}_{m}(R, k)$ replacing $\tilde{B}_{m}(R, k)$. Here $\tilde{B}_{m}(R, k)$ and $\tilde{A}_{m}(R, k)$ are the Fourier transforms of $B_{m}(R, z)$ and $A_{m}(R, z), e . g$.

$$
\tilde{B}_{m}(R, k)=\frac{1}{\sqrt{2 \pi}} \int_{-\infty}^{\infty} d z e^{-i k z} B_{m}(R, z) .
$$

In the case where the magnetic field is produced by an iron dominated magnet, and is therefore localized in space, the integrals (8) can be considered to have, in practice, finite limits of integration. With some care, an effective cutoff can also be found even if the fields extend to infinity since they fall off sufficiently rapidly at infinity. Also, since the generalized Bessel function $I_{m}^{\prime}(w)$ increases exponentially for large $|w|$, there is also, in effect, a cut-off in $k$ for the integral (7) defining the generalized gradients.

\section{TESTS AND EXAMPLES}

The method described in Section 3 has been implemented in the code MARYLIE [2] as a pair of user-defined routines. Versions of the two routines exist for both MARYLIE 3.0, which has recently been released [5] and MARYLIE 5.0, which is still under developement. The first routine, 'usr15', reads the magnetic field data from an external file and computes the functions $A_{m}(R, z)$ and $B_{m}(R, z)$. The second routine, 'usr16', uses the output of 'usr15' to calculate the corresponding tranfer map. In the input file one has to provide a listing of the $B_{x}$ and $B_{y}$ components of the magnetic field together with the coordinates $(x, y, z)$ of the points on the cylindrical surface of radius $\mathrm{R}$ on which the field is defined. For a fixed $z$, there are $n_{\phi}$ equally distributed points along a circumference of radius $R$ centered in $z$ and there are $n_{z}$ such slices. A user-defined routine in MARYLIE is invoked in the master input file like any other MARYLIE command, together with the required parameters. For example, for 'usr15' there are two sets of parameters one needs to specify. The first set consists of the file number containing the magnetic field data, the numbers $n_{\phi}$ and $n_{z}$, a scaling factor for the $z$ coordinate and the magnetic field and the radius $\mathrm{R}$. The second parameter set contains the numbers of files in which to write the normal and skew harmonics, and the values of the integrated harmonics respectively. An excerpt of a MARYLIE master input file containing a setting for 'usr15' is shown below:

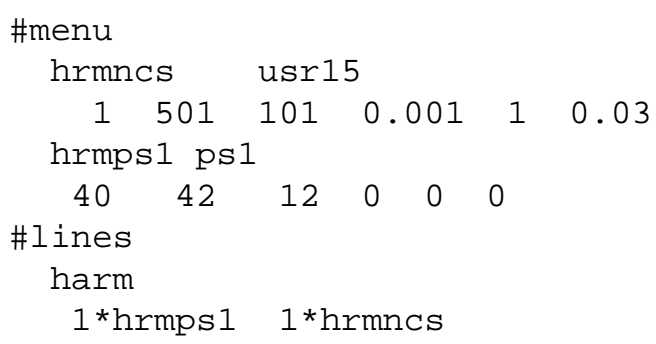

In order to test both the routines and the method we treated the case of an ideal iron-free Lambertson quadrupole. The use of this case as an example has the virtue that the various $C_{m, \alpha}^{[n]}(z)$ can also be determined analytically (see [3]) given a knowldge of the location of the conductors. Results are shown in Figs. 1 and 2. In Fig. 1 the dashed 


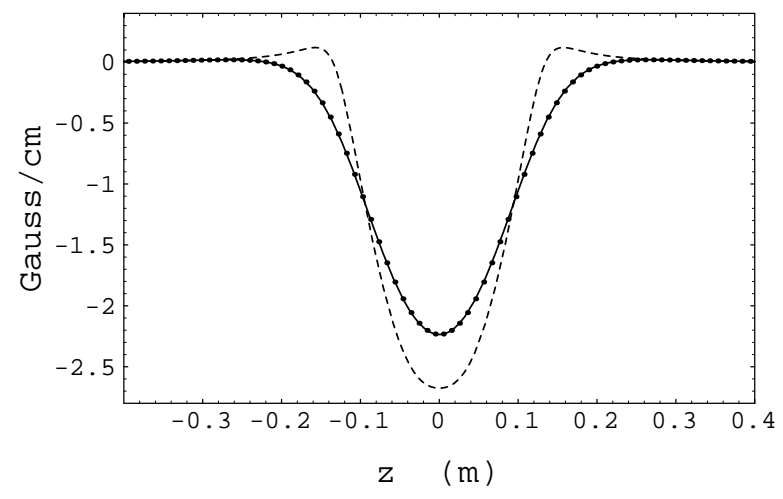

Figure 1: Plot of the scaled harmonic $B_{2}(R, z) / R$ (dashed line) and the on-axis gradient $2 C_{2, s}^{[0]}(z)$ as calculated from surface data (dots) and analytically (solid line) for an ideal Lambertson quadrupole.

line is the function $B_{2}(R, z) / R$ as calculated numerically by using the Biot-Savart law. The solid line represents the on-axis gradient, which is equal to $2 C_{2, s}^{[0]}(z)$, as calculated analytically, while the dots represent the same function as calculated from the surface data. The deviation between $B_{2}(R, z) / R$ and $2 C_{2, s}^{[0]}(z)$ is due to terms in the multipole expansion containing derivatives of $2 C_{2, s}^{[0]}(z)$. These terms are the so called pseudo-multipoles. This can be seen by writing the multipole expansion for $B_{\rho}$ through $6^{\text {th }}$ order in $\rho$ :

$$
\begin{aligned}
B_{\rho} & =\left(2 C_{2, s}^{[0]} \rho-\frac{1}{3} C_{2, s}^{[2]} \rho^{3}+\frac{1}{64} C_{2, s}^{[4]} \rho^{5}\right) \sin 2 \phi \\
& +6 C_{6, s}^{[0]} \rho^{5} \sin 6 \phi .
\end{aligned}
$$

In Fig. 2, as an indication of the reliability of the method, we report the 8th derivative of the generalized gradient (needed for a $9^{\text {th }}$ order code) calculated from the surface data (dots) compared to the anlytical profile (solid line).

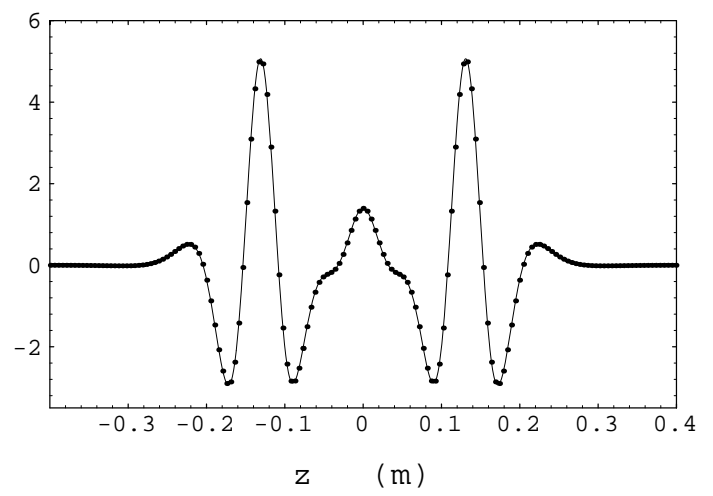

Figure 2: Function $C_{2, s}^{[8]}(z)$ (in units of $10^{-5} \mathrm{Gauss} / \mathrm{cm}^{9}$ ) as calculated from surface data (dots) and analytically (solid line) for an ideal Lambertson quadrupole.

Finally as an example of application, Figs. 3 and 4 show the result of magnetic field analysis for the Return end of a

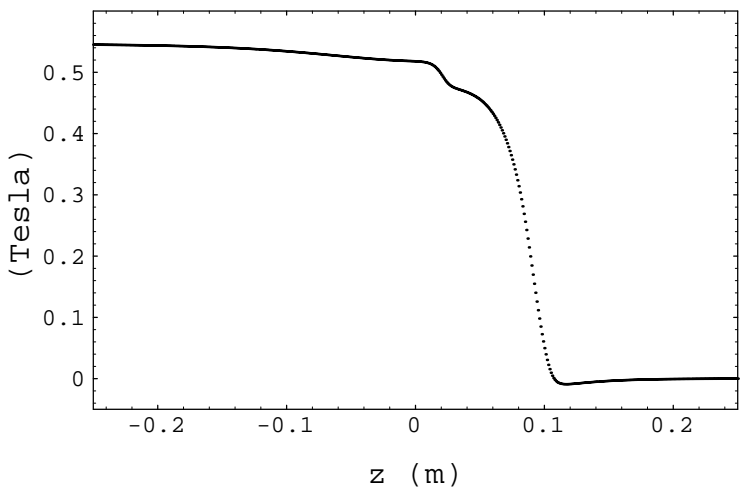

Figure 3: Harmonic $B_{2}(R, z)$ (quadrupole field component) for the Return End; $\mathrm{R}=3 \mathrm{~cm}$.

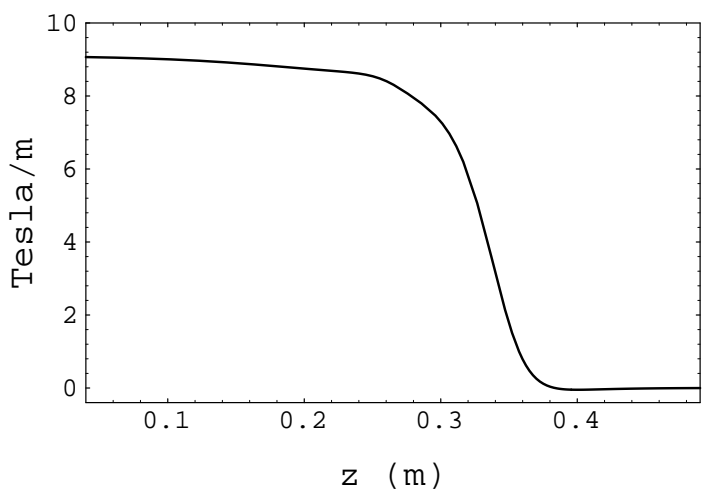

Figure 4: Generalized gradient $C_{2, s}(z)$ for the Return End, ( $z=25 \mathrm{~cm}$ in this picture corresponds to $z=0$ of Figs. 3 ).

High Gradient quadrupole in LHC [6].

\section{ACKOWLEDGEMENTS}

We thank P. Walstrom for helpful discussions and for sharing his Fourier integration and Bessel function routines with us.

\section{REFERENCES}

[1] A. Dragt, Lie Methods for Nonlinear Dynamics with Applications to Accelerator Physics, University of Maryland Physics Department Report (1998).

[2] A. Dragt, F. Neri, G. Rangarajan, D. Douglas, L. Healy, and R. Ryne, Ann. Rev. Nucl. Part. Sci. 38 (1988) pp. 455-96.

[3] M. Venturini and A. Dragt, Accurate Computation of Transfer Maps from Magnetic Field Data, to be published in NIM-A.

[4] S. Caspi, M. Helm, and L.J. Laslett, IEEE Trans. on Magnetics, vol.30, no. 4, (1994).

[5] A. Dragt, Release of MARYLIE 3.0, these proceedings.

[6] G. Sabbi, HGQSO3 End Field Analysis, Fermilab Report TD-98-010 (1998); Private Communication. 